\title{
Magnetic susceptibility of Mn(III) complexes of
}

\section{hydroxamate siderophores}

Stephen D. Springer and Alison Butler*

Department of Chemistry and Biochemistry, University of California, Santa Barbara, CA 931069510, USA

*Corresponding Author: Tel: 805-893-8178. Fax: 805-893-4120. E-mail: butler@chem.ucsb.edu. 
ABSTRACT: The hydroxamate siderophores putrebactin, desferrioxamine B, and desferrioxamine $\mathrm{E}$ bind $\mathrm{Mn}(\mathrm{II})$ and promote the air oxidation of $\mathrm{Mn}(\mathrm{II})$ to $\mathrm{Mn}(\mathrm{III})$ at $\mathrm{pH}>7.1$. The magnetic susceptibility of the manganese complexes were determined by the Evans method and the stoichiometry was probed with electrospray ionization mass spectrometry (ESIMS). The room temperature magnetic moments $\left(\mu_{\mathrm{eff}}\right)$ for the manganese complexes of desferrioxamines B and $\mathrm{E}$ were $4.85 \mathrm{BM}$ and $4.84 \mathrm{BM}$, respectively, consistent with a high spin, $\mathrm{d}^{4}, \mathrm{Mn}(\mathrm{III})$ electronic configuration. The manganese complex of putrebactin had a magnetic moment of 4.98 $\mathrm{BM}$, consistent with incomplete oxidation of $\mathrm{Mn}(\mathrm{II})$, as confirmed by X band EPR spectroscopy. Mass spectra of the $\mathrm{Mn}(\mathrm{III})$ desferrioxamine $\mathrm{B}$ and $\mathrm{E}$ complexes showed complexes at $\mathrm{m} / \mathrm{z}$ 613.26 and 653.26, respectively, consistent with 1:1 complexation. Mass spectral peaks for manganese putrebactin at $\mathrm{m} / \mathrm{z} 797.31$ and 1221.41 corresponds to $1: 2$ and 2:3 Mn:putrebactin complexation. This study directly confirms the $\mathrm{Mn}(\mathrm{III})$ oxidation state in hydroxamate siderophore complexes. 


\section{Introduction}

Siderophores are low molecular weight chelating agents produced by microbes in low iron environments to facilitate iron uptake [1]. In addition to $\mathrm{Fe}(\mathrm{III})$, siderophores have been shown to chelate other hard metal ions, such as $\mathrm{Al}(\mathrm{IIII})$ [2], $\mathrm{Ga}$ (III) [2], $\mathrm{Mo}(\mathrm{VI})$ [3], $\mathrm{Ti}(\mathrm{IV})$ [4], and $\mathrm{V}(\mathrm{V})[3,5,6]$, among others. Manganese is of particular interest because it is often found in the environment in oxide minerals. In a similar fashion to siderophore-mediated extraction of Fe(III) from iron oxide minerals, siderophores have been shown to dissolve manganite $(\gamma-$ $\mathrm{MnOOH})$ and hausmannite $\left(\mathrm{Mn}_{3} \mathrm{O}_{4}\right)$ through $\mathrm{pH}$-dependent processes [7-9]. Thus siderophores may be involved in biogenic cycling of manganese [10-12]. $\mathrm{Mn}(\mathrm{III})$ is not stable in aqueous solutions and readily disproportionates to $\mathrm{Mn}(\mathrm{II})$ and a variety of $\mathrm{Mn}(\mathrm{IV})$ oxides and hydroxides [13]. However $\mathrm{Mn}$ (III) can form stable complexes in aqueous solutions with oxygen donating ligands, including hydroxamates $[14,15]$.

$\mathrm{Mn}(\mathrm{III})$ complexes of the linear and cyclic tris-hydroxamate siderophores, desferrioxamine B (DFOB) and desferrioxamine E (DFOE), respectively, were first investigated as mimics of superoxide dismutase [16-18]. Upon complexation of Mn(II) by DFOB or DFOE, air oxidation of $\mathrm{Mn}(\mathrm{II})$ under basic conditions ( $\mathrm{pH}$ 8.5) produced a clear green solution [19], similar to the complex formed on addition of $\mathrm{Mn}(\mathrm{OH})_{3}$ to DFOB and DFOE, as well as on addition of $\mathrm{MnO}_{2}$ to DFOB and DFOE in the presence of a reductant [16]. Duckworth and Sposito found that the rate of oxidation of $\mathrm{Mn}$ (II)-DFOB by $\mathrm{O}_{2}$ is fastest in the fully deprotonated complex $[7,19]$. They also found that the proton-independent stability constant of $\mathrm{Mn}(\mathrm{III})-\mathrm{DFOB}$ is remarkably high, at $\log K 29.9( \pm 0.5)$ [19], and approaches that of Fe(III)DFOB at $\log K 30.5[20]$. 
To further characterize the $\mathrm{Mn}(\mathrm{III})$ complexes of hydroxamate siderophores, we have measured the magnetic susceptibility of the air-oxidized $\mathrm{Mn}(\mathrm{II})$-siderophore complexes to directly probe the Mn(III) oxidation state in aqueous solution, using the Evans NMR technique [21-23]. EPR spectroscopy was also employed to detect if any $\mathrm{Mn}(\mathrm{II})$ remained. We selected three structural hydroxamate archetypes for this study: the linear and cyclic tris-hydroxamate siderophores, DFOB, DFOE, and the cyclic bis-hydroxamate siderophore, putrebactin (Figure1). Comparison of the tris-hydroxamate to bis-hydroxamate siderophores is of particular interest due to differences expected in the stoichiometry of coordination, analogous to the Fe(III)-siderophore complexes [24-27]. Possible coordination complexes of $\mathrm{Mn}$ (III) and putrebactin could include the neutral bridged 2:3 Mn-putrebactin or 1:2 Mn-putrebactin complexes shown in Figure 2, as well as multiple 1:1 Mn-putrebactin aqua or hydroxo complexes, or possibly 2:2 Mn-putrebactin $\mu$-oxo or $\mu$-hydroxo dimers (Figure 2).

\section{Figure 1.}

\section{Figure 2.}

\section{Experimental}

2.1. General experimental procedures. All UV-visible (UV-Vis) spectrophotometry was carried out on a Varian Cary-Bio 300 UV-Vis spectrophotometer. ${ }^{1} \mathrm{H}$ NMR spectra were recorded on a Varian Unity Inova $400 \mathrm{MHz}$ spectrometer (5 $\mathrm{mm}$ broad band probe) at room temperature. Mass spectra were obtained using electrospray ionization mass spectrometry (ESIMS) and tandem mass spectrometry (ESI-MS/MS) on a Micro Mass QTOF-2 mass spectrometer (Waters Corp.), using argon as the collision gas. Inductively coupled plasma atomic emission spectroscopy (ICP-AES), measurements were taken on a Thermo iCAP 6300, and calibrated 
from dilutions of $1000 \mathrm{ppm} \mathrm{Mn}$ (II) or $\mathrm{Fe}(\mathrm{III})$ standard solutions (Fisher), using class A volumetric glassware. X-Band EPR spectra were obtained using a Bruker EMXplus Spectrometer with an aqueous flat cell.

2.2 Siderophores: DFOB, DFOE and putrebactin. DFOB mesylate was purchased from Sigma-Aldrich and used as obtained.

Isolation of putrebactin. Putrebactin was isolated from cultures of Shewanella oneidensis MR1.[26] S. oneidensis MR-1 was maintained on Difco 2216 marine broth plates, and cultured in an artificial sea water medium (ASG+Fe), containing $2 \mathrm{~L}$ doubly deionized water, $20 \mathrm{~g}$ Bacto CAS amino acids, 2 g NH $\mathrm{H}_{4} \mathrm{Cl}, 2 \mathrm{~g}$ glycerol phosphate, $24.7 \mathrm{~g} \mathrm{MgSO}_{4} \cdot 7 \mathrm{H}_{2} \mathrm{O}, 2.9 \mathrm{~g} \mathrm{CaCl}_{2} \cdot \mathrm{H}_{2} \mathrm{O}, 33.1 \mathrm{~g}$ $\mathrm{NaCl}, 1.5 \mathrm{~g} \mathrm{KCl}, 6 \mathrm{~mL}$ glycerol, and $2 \mu \mathrm{M}$ (final concentration) of $\mathrm{FeCl}_{3}$. Prior to inoculation, 20 $\mathrm{mL}$ of filter-sterilized 1.0 M HEPES buffer (pH 7.4), $4 \mathrm{~mL}$ of filter-sterilized 1.0 $\mathrm{M} \mathrm{NaHCO}_{3}$ buffer, and $20 \mathrm{~mL}$ filter-sterilized vitamin stock were added to the medium. Vitamin stock consisted of $4 \mathrm{mg}$ niacin, $2 \mathrm{mg}$ thiamin, $4 \mathrm{mg} p$-aminobenzoic acid, $2 \mathrm{mg}$ calcium pantothenic acid, $20 \mathrm{mg}$ pyridoxine $\mathrm{HCl}, 2 \mathrm{mg}$ cyanocobalamin, $4 \mathrm{mg}$ riboflavin, and $4 \mathrm{mg}$ folic acid in 200 $\mathrm{mL}$ doubly deionized water.

Isolation of DFOE. DFOE was obtained from an uncharacterized bacterium isolated from seawater obtained from the Gulf of Mexico after the Deepwater Horizon oil spill in 2010; the bacterium was maintained on minimal nutrient seawater agar plates consisting of $0.5 \mathrm{~g}$ Bacto yeast extract, $5 \mathrm{~g}$ Bacto peptone, $15 \mathrm{~g}$ Bacto agar in $1 \mathrm{~L}$ filtered seawater. The bacterium was cultured in a minimal nutrient artificial seawater medium (ASW-Fe) composed of $30 \mathrm{~g} \mathrm{NaCl}$, $1.5 \mathrm{~g} \mathrm{KCl}, 6 \mathrm{~g} \mathrm{CaCl}_{2} \cdot 2 \mathrm{H}_{2} \mathrm{O}, 24 \mathrm{~g} \mathrm{MgSO}_{4} \cdot 7 \mathrm{H}_{2} \mathrm{O}, 2 \mathrm{~g} \mathrm{NH}_{4} \mathrm{Cl}, 0.2$ g glycerol phosphate, 4 g Bacto CAS amino acids, and $6 \mathrm{~mL}$ glycerol in $2 \mathrm{~L}$ doubly deionized water (Barnstead Nanopure II). 



sterilized $1.0 \mathrm{M} \mathrm{NaHCO}_{3}$ buffer were added to the medium.

For siderophore isolation, each bacterial strain was grown for $24-48 \mathrm{~h}$ on an orbital shaker $(180 \mathrm{rpm})$ and harvested via centrifugation. Purification of putrebactin and DFOE followed the same general procedure. Amberlite XAD-2 resin (Supelco) was combined with the cell free culture supernatant $(100 \mathrm{~g} / \mathrm{L})$ and shaken for $2 \mathrm{~h}(120 \mathrm{rpm})$. Supernatant was then filtered away and the XAD-2 resin was rinsed with doubly deionized water $(1 \mathrm{~L} / 100 \mathrm{~g})$, prior to packing the XAD-2 resin into a glass chromatography column $(2 \mathrm{~cm}$ internal diameter). Siderophores were eluted in $100 \%$ methanol. The eluent was lyophilized, and stored at stored at $20{ }^{\circ} \mathrm{C}$.

In the isolation procedure for putrebactin, the resulting yellow brown powder was rinsed approximately five times with ca. $10 \mathrm{~mL}$ doubly deionized water until the solid became white. The white solid was re-dissolved in $300 \mathrm{~mL} \mathrm{MeOH}$ with gentle heating, at which point $25 \mathrm{~mL}$ of $\mathrm{H}_{2} \mathrm{O}$ was added, followed by rotary evaporation to remove $\mathrm{MeOH}$. Final purification via RPHPLC was performed on a $\mathrm{C}_{4}$ preparatory column $(10 \mu \mathrm{m}$ particle size, $250 \mathrm{~mm}$ column length, x $20 \mathrm{~mm}$ i.d., Higgins Analytical). A gradient of $100 \% \mathrm{H}_{2} \mathrm{O}$ (doubly deionized with $0.05 \%$ trifluoric acetic acid (TFA) to $35 \% \mathrm{MeOH}(0.05 \% \mathrm{TFA})$ over 30 min was used for putrebactin, and a $100 \% \mathrm{H}_{2} \mathrm{O}$ to $80 \% \mathrm{MeOH}$ gradient $(0.05 \%$ TFA) over 40 min was used for DFOE. The eluent was monitored continuously at $215 \mathrm{~nm}$ and putrebactin-containing fractions, as confirmed by mass spectrometry, were pooled, lyophilized and stored at $-20^{\circ} \mathrm{C}$.

To analyze for adventitious iron contamination in the siderophore samples, the siderophore ligands (final concentration $0.1 \mathrm{mM}$ ) were dissolved in $1 \mathrm{~mL}$ of nitric acid $(70 \%)$ 
and diluted into $100 \mathrm{~mL}$ in doubly de-ionized water. The ICP-AES spectrometer was calibrated in the linear range using a blank of $0.7 \%$ nitric acid and a $0.1 \mathrm{ppm}$ Fe solution in $0.7 \%$ nitric acid prepared by dilution of a 1000 ppm Fe(III) standard (Fisher). Each sample was run in triplicate with an $80 \mathrm{~s}$ exposure time.

2.3. Formation of Mn(III)-siderophore complexes. A stock solution of $10 \mathrm{mM} \mathrm{Mn}(\mathrm{II})$ was prepared from $\mathrm{MnCl}_{2} \bullet 4 \mathrm{H}_{2} \mathrm{O}$ in $\mathrm{D}_{2} \mathrm{O}(99.9 \%)$ and standardized by ICP-AES. Mn(II) (1 mM final concentration) was reacted in air with $2 \mathrm{mM}$ DFOB or DFOE and $3 \mathrm{mM}$ putrebactin, in water at $\mathrm{pH}$ 8-10 (or up to $\mathrm{pH} 9.5$ for putrebactin) for 24 hrs. To adjust the $\mathrm{pH}, \sim 1 \mathrm{M} \mathrm{NaOD}$ or DCl was added, as needed. The $\mathrm{NaOD}$ and $\mathrm{DCl}$ solutions were prepared by dissolving solid $\mathrm{NaOH}$ and concentrated $\mathrm{HCl}(36 \%)$ into $\mathrm{D}_{2} \mathrm{O}(99.9 \%)$. Solubilization of putrebactin in water required addition of $\mathrm{NaOD}$ to maintain the $\mathrm{pH}$ above 7.1. A 6 inch micro combination electrode (Accumet) with a calomel reference was used to monitor the $\mathrm{pH}$ of the solutions. The oxidation of $\mathrm{Mn}$ (II) to $\mathrm{Mn}$ (III) was followed at $310 \mathrm{~nm}$ which is in the range of the $\lambda_{\max }$ for the $\mathrm{Mn}$ (III) siderophore complexes. Solutions were filtered for EPR spectroscopic analysis and magnetic susceptibility measurements. The solutions for EPR spectroscopy and UV-Vis analysis were buffered by $50 \mathrm{mM}$ CAPSO ( $N$-cyclohexyl-2-hydroxyl-3-aminopropanesulfonic acid) at $\mathrm{pH} 9$.

2.4. Magnetic Susceptibility. The effective magnetic moment $\left(\mu_{\mathrm{eff}}\right)$ of the Mn-siderophore complexes was determined at room temperature using the Evans NMR method [23, 28]. DMSO $(10 \% \mathrm{v} / \mathrm{v})$ was used for the reference. The initial concentration of $\mathrm{Mn}(\mathrm{II})(1 \mathrm{mM})$ was used for calculation purposes allowing for comparison between siderophore ligands, independent of the Mn(III)-complex stoichiometry. Calculation of the molar magnetic susceptibility $\left(\chi_{\mathrm{M}}\right)$ was performed using a modified Evans equation [28, 29] which takes into account the geometry of superconducting magnets (Equation 1). In equation $1, \chi_{D}$ is the diamagnetic correction for the 
ligands, $\mathrm{M}$ is molarity, $\mathrm{m}$ is mass, $\Delta f$ is the chemical shift, and $f_{\mathrm{o}}$ is the operating frequency [28, 29]. The $\mu_{\text {eff }}$ was calculated from equation 2 .

$$
\begin{aligned}
& \chi_{M}=\frac{750 \Delta f}{\pi f_{o} M}+\chi_{D} \\
& \mu_{e f f}=2.828 \sqrt{\chi_{M} T}
\end{aligned}
$$

2.5. Mass spectrometry. Samples for mass spectroscopy were prepared using stoichiometric amounts of siderophore and $\mathrm{Mn}(\mathrm{II})$. The $\mathrm{pH}$ was adjusted to $\mathrm{pH} 8-10$ with $1 \mathrm{M} \mathrm{NH}_{4} \mathrm{OH}$. The mass spectrometer was run in positive ion mode with a flow rate of $10 \mu \mathrm{L} / \mathrm{sec}$. Tandem mass spectroscopy was used to confirm the identity of the ligands used.

2.6. EPR Spectroscopy. Samples for EPR analysis were prepared in $\mathrm{D}_{2} \mathrm{O}(99.9 \%)$ with $10 \% \mathrm{v} / \mathrm{v}$ DMSO. X-band EPR spectra $(9.78 \mathrm{GHz})$ were obtained using an aqueous flat cell at room temperature to detect residual $\mathrm{Mn}(\mathrm{II})$ after air oxidation of the $\mathrm{Mn}(\mathrm{II})$-siderophore complexes.

\section{Results and Discussion}

3.1. Magnetic susceptibility of Mn(III) complexes of DFOB, DFOE and putrebactin. The manganese complexes for magnetic susceptibility measurements were prepared, as described above, by reacting $2 \mathrm{mM}$ DFOB or DFOE with $1 \mathrm{mM} \mathrm{Mn}(\mathrm{II})$ or $3 \mathrm{mM}$ putrebactin with $1 \mathrm{mM}$ $\mathrm{Mn}(\mathrm{II})$ in $99.9 \% \mathrm{D}_{2} \mathrm{O}$, followed by exposure to air for $24 \mathrm{hr}$, and after adjustment to $\mathrm{pH} 8-10$. Upon siderophore complexation, $\mathrm{Mn}(\mathrm{II})$ oxidation occurs within a $\mathrm{pH}$ range of 7.1-11.1, a range established in previous investigations on the formation of $\mathrm{Mn}(\mathrm{III})$-DFOB.[19] The extinction coefficient of $2062 \mathrm{M}^{-1} \mathrm{~cm}^{-1}$ at $310 \mathrm{~nm}$ was measured for $\mathrm{Mn}(\mathrm{III})-\mathrm{DFOB}$, in agreement with previously reported values, and used to monitor the extent of the Mn(III)-DFOB formation.[16] 
DFOE had a similar $\mathrm{pH}$ dependency and UV-Vis spectrum as DFOB (Supplementary Information Figure S1).

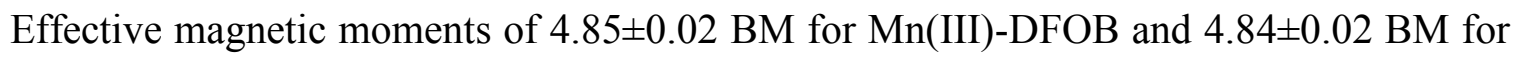
Mn(III)-DFOE were obtained using the Evans NMR method for these complexes in aqueous solution (Table S1). The magnetic moment values agree well with the calculated value of 4.90 $\mathrm{BM}$ for a $\mathrm{d}^{4}$ high spin ion and can be used as a baseline for comparison to other hydroxamate siderophores.

The magnetic moment resulting from reaction of $1 \mathrm{mM} \mathrm{Mn}(\mathrm{II})$ and $3 \mathrm{mM}$ putrebactin in air for $24 \mathrm{~h}$ and up to $\mathrm{pH} 9.5$ was $4.98 \pm 0.02 \mathrm{BM}$ (Table S1), which is higher than the calculated magnetic moment of $4.90 \mathrm{BM}$, assuming complete oxidation of $\mathrm{Mn}$ (II) to $\mathrm{Mn}$ (III) and higher than the Mn(III)-DFOB and Mn(III)-DFOE complexes. The value of the magnetic moment in the Mn-putrebactin sample was unexpected and iron contamination was suspected. However ICP-AES analysis of the putrebactin samples showed no significant level of iron was present (Table S2).

To investigate whether the higher magnetic moment resulted from incomplete oxidation of $\mathrm{Mn}(\mathrm{II})$, the X-band EPR spectrum was examined. High spin $\mathrm{d}^{5} \mathrm{Mn}(\mathrm{II})$ has a characteristic 6 peak spectrum at room temperature $[30,31]$, whereas high spin $\mathrm{d}^{4} \mathrm{Mn}(\mathrm{III})$ is EPR silent. EPR analysis of the Mn-putrebactin reaction solution gave a weak 6 line signal (Figure 3), which matches the control EPR spectrum for aqueous Mn(II). Assuming that the only species contributing to the magnetic susceptibility were high spin $\mathrm{Mn}(\mathrm{II})$ and $\mathrm{Mn}(\mathrm{III})$, the observed $\mu_{\mathrm{eff}}$ of 4.98 for the Mn-putrebactin solution is consistent with the presence of $7.8 \% \mathrm{Mn}(\mathrm{II})$. The EPR spectra of the Mn(III)-DFOE and Mn(III)-DFOB did not show residual Mn(II) (Figures S2-S4). 


\section{Figure 3.}

Efforts to increase the extent of $\mathrm{Mn}(\mathrm{II})$ oxidation in the putrebactin reactions at elevated pH was hampered by precipitation above $\mathrm{pH} 9.5$, which differs from the stability of $\mathrm{Mn}$ (III)DFOB and Mn(III)-DFOE up to $\mathrm{pH} 11.1$. The second $\mathrm{pK}_{\mathrm{a}}$ of putrebactin at 9.71 [26] is higher than the stable solution $\mathrm{pH}$ for the $\mathrm{Mn}(\mathrm{II} / \mathrm{III})$-putrebactin complexes, which may contribute to the lack of formation and stabilization of $\mathrm{Mn}(\mathrm{III})$-putrebactin complexes, since the Mn(III) oxidation state is stabilized by complexation to hard anionic ligands.

3.2. Mass spectrometry of the Mn(III) complexes of DFOB, DFOE and putrebactin. Mass spectra for the manganese complexes of DFOB and DFOE showed masses at m/z 613.26 and 653.26, respectively, corresponding to $1: 1 \mathrm{Mn}(\mathrm{III})$-siderophore complexes, as expected for trishydroxamate siderophores (Figures S5, S6). Because putrebactin is a bis-hydroxamate ligand, it is not expected to exhibit the 1:1 metal to ligand complexation typical of tris-hydroxamate siderophores. Previous studies with Fe(III) and the bis-hydroxamate siderophore, alcaligin (Figure $1, \mathrm{R}=\mathrm{OH}$ ) $[24,25,32,33]$, revealed $\mathrm{pH}$-dependent speciation with 1:1, 2:3, and 2:2 $\left[\mathrm{Fe}(\mathrm{III})_{\mathrm{x}}\right.$-alcaligin $\left.\mathrm{y}\right]$ complexes at low $(\mathrm{pH}<5)$, mid $(\mathrm{pH} 5-9)$, and high $(\mathrm{pH}>9) \mathrm{pH}$ values respectively [25]. The 2:2 complex formed at $\mathrm{pH} 11$ is reported to be an anti-ferromagnetically coupled bis- $\mu$-oxo dimer, $\mathrm{Fe}_{2}$ (alcaligin) $)_{2} \mathrm{O}_{2},[25]$ and not detected at lower $\mathrm{pH}$ by ESI-MS [33].

Mass spectrometry was used to explore the speciation present in solutions resulting from air oxidation of $1 \mathrm{mM} \mathrm{Mn}(\mathrm{II})$ in the presence of $1.5 \mathrm{mM}$ putrebactin, $\mathrm{pH} 9.5$. The peaks at $\mathrm{m} / \mathrm{z}$ 797.3 and 1221.4 (Figure 4A) are consistent with 1:2 and 2:3 Mn-putrebactin complexes, [Mn(putrebactin $\left.)_{2}+\mathrm{H}^{+}\right]$and $\left[\left(\mathrm{Mn}_{2}-\right.\right.$ putrebactin $\left.\left._{3}\right)+\mathrm{H}^{+}\right]$, respectively. The peaks at $\mathrm{m} / \mathrm{z} 425.1$ and 850.2 appear to be fragmentation products of the $1: 2(\mathrm{~m} / \mathrm{z} 797.3)$ and $2: 3(\mathrm{~m} / \mathrm{z} 1221.4)$ complexes, 
confirmed by tandem mass spectrometry (Figures 4B, 4C), and in each case corresponds to the loss of a putrebactin ligand.

\section{Figure 4.}

The 1:2 ratio of metal(III)-siderophore complexation has previously been reported for $\mathrm{Fe}(\mathrm{III})$ and alcaligin when the siderophore is present in excess [33]. Thus for the reaction of 1 $\mathrm{mM} \mathrm{Mn}(\mathrm{II})$ and $1.5 \mathrm{mM}$ putrebactin, it is not surprising to observe species of $\mathrm{Mn}_{2}$-putrebactin 3 or Mn-putrebactin 2 . Evidence for a $\mathrm{Mn}(\mathrm{III}) \mu$-oxo-dimer, analogous to the related $\mathrm{Fe}(\mathrm{III})$ complex, $\mathrm{Fe}_{2}$ (alcaligin) $)_{2} \mathrm{O}_{2}$ [25], was not detected by negative ion mode mass spectrometry (Figure S7). Additionally, the 1:1 Mn(III)-putrebactin complex was not observed. Metal to ligand stoichiometries of 1:1 for Fe(III)-bis-hydroxamate complexes are normally found below $\mathrm{pH} 5$ [25], however oxidation of $\mathrm{Mn}$ (II) does not occur below $\mathrm{pH} 7.1$, possibly explaining the lack of evidence for this species.

\section{Conclusions}

In sum, the magnetic susceptibility determination of the reaction products of the aerobic reactions of $\mathrm{Mn}(\mathrm{II})$ and the hydroxamate siderophores, $\mathrm{DFOB}, \mathrm{DFOE}$ and putrebactin, directly confirm the Mn(III) siderophore complex is formed quantitatively with DFOB and DFOE (pH 810). In the $\mathrm{Mn}(\mathrm{II})$ putrebactin reactions, $\mathrm{Mn}(\mathrm{III})$ formation was nearly stoichiometric however approximately $8 \% \mathrm{Mn}(\mathrm{II})$ remained as detected by EPR spectroscopy and the magnetic moment. Attempts to increase the extent of aerobic oxidation of the Mn(II) putrebactin complexes was hampered by precipitation at $\mathrm{pH}>9.5$. Mass spectrometry showed the presence of two Mn-

putrebactin species, consistent with $\quad \mathrm{Mn}(\mathrm{III})_{2}$ (putrebactin) $)_{3}$ and $\mathrm{Mn}(\mathrm{III})($ putrebactin)(putrebactinH), which is analogous to $\mathrm{Fe}(\mathrm{III})$ complexes of alcaligin above 
neutral $\mathrm{pH}$ [25]. These results complement previous reports on $\mathrm{Mn}$ (III)-DFOB and $\mathrm{Mn}(\mathrm{III})$ DFOE [16].

Siderophores could play a role in increasing the availability of manganese to various organisms. For example, DFOB has been shown to dissolve manganite $(\gamma-\mathrm{Mn}(\mathrm{III}) \mathrm{OOH})$, hausmannite $\left(\mathrm{Mn}_{3} \mathrm{O}_{4}\right)$ and $\mathrm{Mn}(\mathrm{IV})$-type oxides through both reductive and non-reductive pathways, depending on $\mathrm{pH}$ [7]. Thus a pool of Mn(III) chelated by siderophores could increase the availability of manganese to organisms that might not otherwise have a means to obtain it. While ligand-mediated uptake of metals other than iron has been demonstrated recently for molybdenum and vanadium with siderophores [3, 34] and for copper with chalkophores [35, 36], ligand-mediated uptake of manganese has not been reported to date. Nevertheless, siderophores may play an important role in biogenic cycling of manganese $[10,19,37,38]$.

\section{ACKNOWLEDGMENT}

Funding from NSF CHE-1411941 (AB) and NSF DMR-1121053 (UCSB MRL) is gratefully acknowledged. We thank J. Pavlovich (MS) and H. Zhou (NMR) at UCSB for their technical assistance. 


\section{REFERENCES}

[1] M. Sandy, A. Butler, Chemical Reviews, 109 (2009) 4580-4595.

[2] L.D. Loomis, K.N. Raymond, Inorganic Chemistry, 30 (1991) 906-911.

[3] J.P. Bellenger, T. Wichard, A.B. Kustka, A.M.L. Kraepiel, Nature Geosci, 1 (2008) 243-246.

[4] K.M. Buettner, A.M. Valentine, Chemical Reviews, 112 (2012) 1863-1881.

[5] A.A.H. Pakchung, C.Z. Soe, T. Lifa, R. Codd, Inorganic Chemistry, 50 (2011) 5978-5989.

[6] C.Z. Soe, A.A.H. Pakchung, R. Codd, Inorganic Chemistry, 53 (2014) 5852-5861.

[7] O.W. Duckworth, G. Sposito, Environ. Sci. Technol., 39 (2005) 6045-6051.

[8] O.W. Duckworth, G. Sposito, Chemical Geology, 242 (2007) 497-508.

[9] J. Pena, O.W. Duckworth, J.R. Bargar, G. Sposito, Geochimica Et Cosmochimica Acta, 71 (2007) 5661-5671.

[10] O.W. Duckworth, J.R. Bargar, G. Sposito, BioMetals, 22 (2009) 605-613.

[11] M.M. Akafia, J.M. Harrington, J.R. Bargar, O.W. Duckworth, Geochimica Et Cosmochimica Acta, 141 (2014) 258-269.

[12] S. Kraemer, O. Duckworth, J. Harrington, W.C. Schenkeveld, Aquatic Geochemistry, 2014, 10.1007/s10498-014-9246-7.

[13] J.J. Morgan, Geochim. Cosmochim. Acta, 69 (2005) 35-48.

[14] J.M. Harrington, D.L. Parker, J.R. Bargar, A.A. Jarzecki, B.M. Tebo, G. Sposito, O.W. Duckworth, Geochim. Cosmochim. Acta, 88 (2012) 106-119.

[15] S.D. Springer, A. Butler, Coord. Chem. Rev. 2015, http://dx.doi.org/10.1016/j.ccr.2015.03.013

[16] K.M. Faulkner, R.D. Stevens, I. Fridovich, Arch. Biochem. Biophys., 310 (1994) 341-346.

[17] W.F. Beyer, Jr., I. Fridovich, Arch. Biochem. Biophys., 271 (1989) 149-156. 
[18] D. Darr, K.A. Zarilla, I. Fridovich, Arch. Biochem. Biophys., 258 (1987) 351-355.

[19] O.W. Duckworth, G. Sposito, Environ. Sci. Technol., 39 (2005) 6037-6044.

[20] K.N. Raymond, E.A. Dertz, in: J.H. Crosa, A.R. May, S.M. Payne (Eds.) Iron Transport in Bacteria, ASM Press, 2004, pp 3-17.

[21] D.F. Evans, T.A. James, Dalton Trans., (1979) 723-726.

[22] Y. Sheng, T.A. Stich, K. Barnese, E.B. Gralla, D. Cascio, R.D. Britt, D.E. Cabelli, J.S.

Valentine, J. Am. Chem. Soc., 133 (2011) 20878-20889.

[23] D.F. Evans, J. Chem. Soc., (1959) 2003-2005.

[24] Z. Hou, C.J. Sunderland, T. Nishio, K.N. Raymond, J. Am. Chem. Soc., 118 (1996) 51485149 .

[25] Z. Hou, K.N. Raymond, B. O'Sullivan, T.W. Esker, T. Nishio, Inorg. Chem., 37 (1998) 6630-6637.

[26] K.M. Ledyard, A. Butler, J. Biol. Inorg. Chem., 2 (1997) 93-97.

[27] S.J. Barclay, P.E. Riley, K.N. Raymond, Inorg. Chem., 23 (1984) 2005-2010.

[28] S.K. Sur, J. Magn. Reson., 82 (1989) 169-173.

[29] G.A. Bain, J.F. Berry, J. Chem. Educ., 85 (2008) 532-536.

[30] D.P. Goldberg, J. Telser, J. Krzystek, A.G. Montalban, L.-C. Brunel, A.G.M. Barrett, B.M.

Hoffman, J. Am. Chem. Soc., 119 (1997) 8722-8723.

[31] S. Un, A. Sedoud, Appl. Magn. Reson., 37 (2009) 247-256.

[32] H. Boukhalfa, T.J. Brickman, S.K. Armstrong, A.L. Crumbliss, Inorg. Chem., 39 (2000) $5591-5602$.

[33] I. Spasojevic, H. Boukhalfa, R.D. Stevens, A.L. Crumbliss, Inorg. Chem., 40 (2001) 49-58. 
[34] J.P. Bellenger, T. Wichard, A.M.L. Kraepiel, Appl. Environ. Microbiol., 74 (2008) 14781484.

[35] G.E. Kenney, A.C. Rosenzweig, ACS Chem. Biol., 7 (2012) 260-268.

[36] R. Balasubramanian, G.E. Kenney, A.C. Rosenzweig, J. Biol. Chem., 286 (2011) 3731337319.

[37] T.G. Spiro, J.R. Bargar, G. Sposito, B.M. Tebo, Acc. Chem. Res., 43 (2010) 2-9.

[38] O.W. Duckworth, J.R. Bargar, G. Sposito, Geochim. Cosmochim. Acta, 72 (2008) 33713380. 
Figure Legends

Figure 1. Desferrioxamine B (DFOB), desferrioxamine E (DFOE) and putrebactin.

Figure 2. Possible coordination modes of Mn(III) putrebactin complexes.

Figure 3. X-Band EPR spectrum of the $\mathrm{Mn}(\mathrm{III})$-putrebactin complex ( $\left.\mu_{\text {eff }}, 5.04\right)$ in $\mathrm{D}_{2} \mathrm{O}(99.9 \%)$ with $10 \%(\mathrm{v} / \mathrm{v})$ DMSO in an aqueous flat cell at room temperature. Frequency $9.78 \mathrm{GHz}$.

Figure 4. (A) ESI-MS of the reaction mixture of $1 \mathrm{mM} \mathrm{Mn}$ (II) and $1.5 \mathrm{mM}$ putrebactin after aqueous aerobic reaction for $24 \mathrm{~h}, \mathrm{pH}$ 9.1. (B) $\mathrm{ms}-\mathrm{ms}$ of $\mathrm{m} / \mathrm{z} 1221.41$ in (A) with $\mathrm{m} / \mathrm{z} 850.24$ as the only fragmentation product. (C) ms-ms of m/z 797.30 in (A) with $425.14 \mathrm{~m} / \mathrm{z}$ as the only fragmentation product. 
Figure 1.

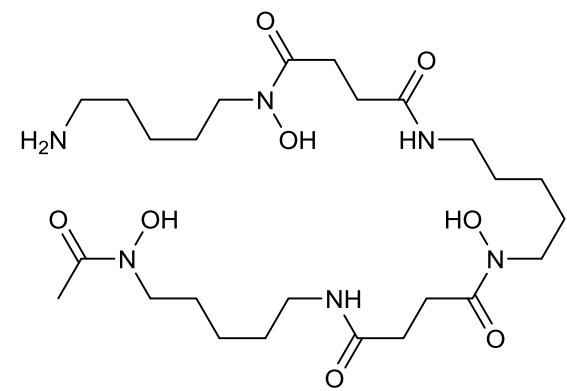

DFOB

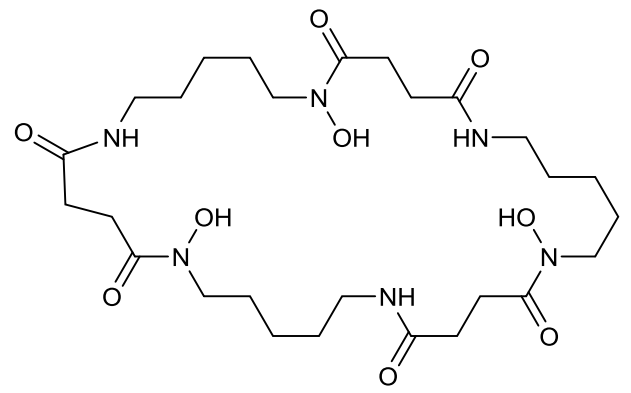

DFOE



putrebactin, $\mathrm{R}=\mathrm{H}$

Figure 2.
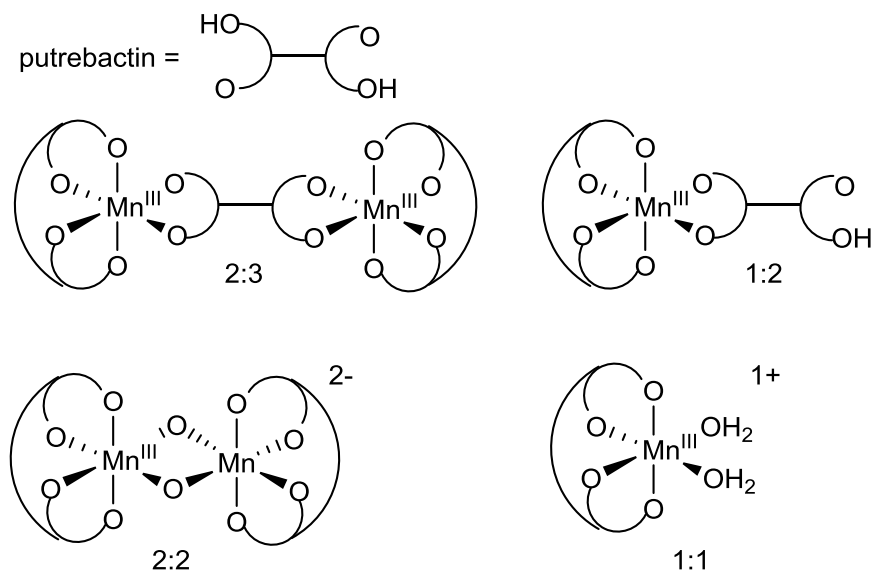
Figure 3.

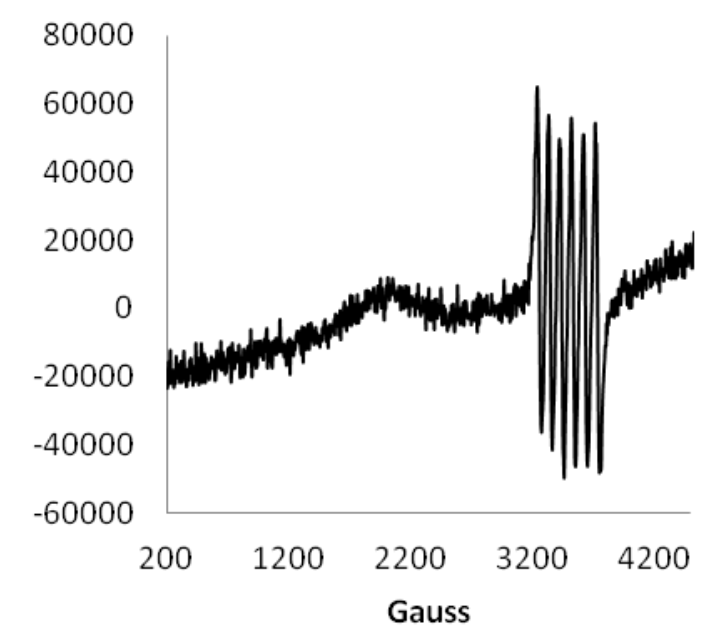


Figure 4.

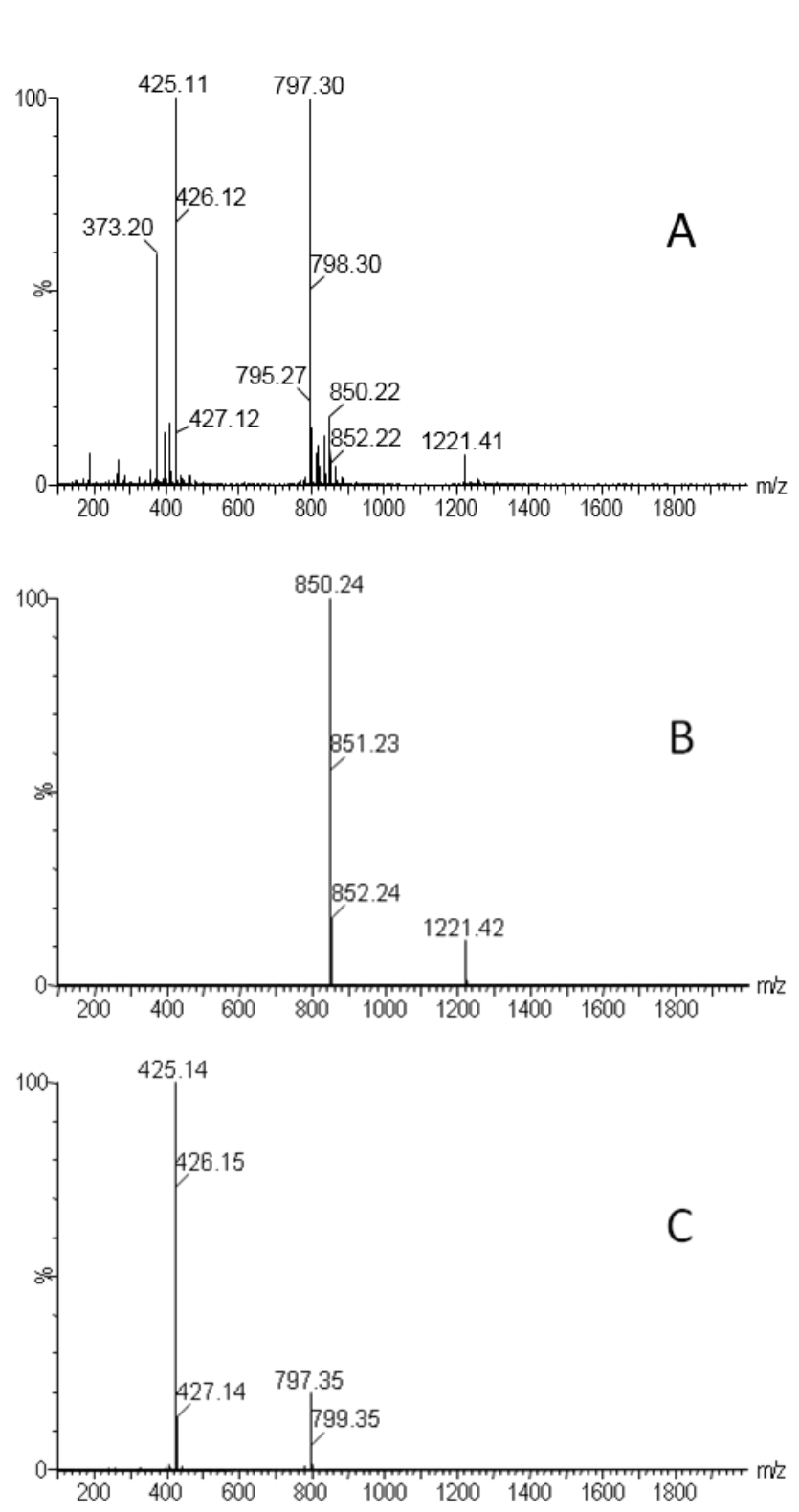



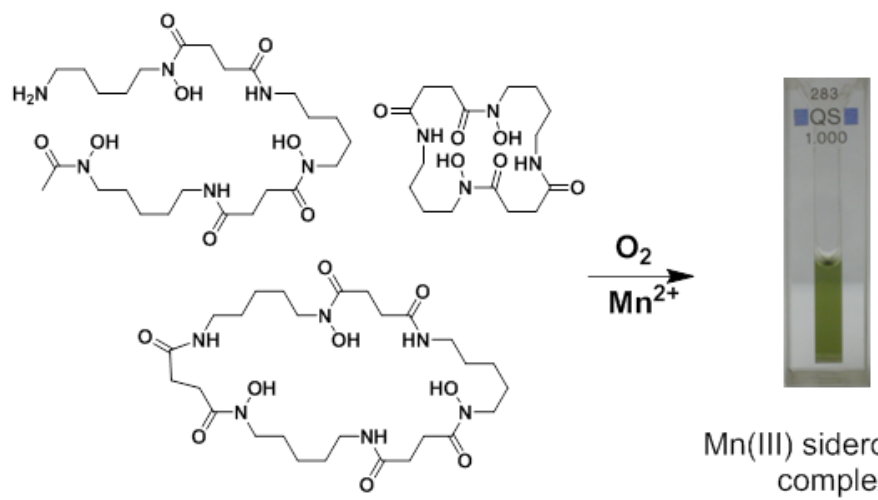

$\mathrm{Mn}$ (III) siderophore complex

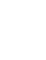

